\section{G290 THE DEVELOPMENT OF A PAEDIATRIC HEALTHCARE AT HOME SERVICE}

${ }^{1} \mathrm{RC}$ Mitchell, ${ }^{2} \mathrm{Z}$ Tribble, ${ }^{1} \mathrm{~L}$ Watt, ${ }^{1} \mathrm{~S}$ Harris, ${ }^{1} \mathrm{M}$ Beardsmore-Rust, ${ }^{1} \mathrm{O}$ Akindolie, ${ }^{1}$ Department of Ambulatory Paediatrics, Kings College Hospital NHS Foundation Trust, London, UK; ${ }^{2}$ Healthcare at Home

10.1136/archdischild-2015-308599.267

Aims The 2012 NHS mandate recommended reduction in the length of stay for inpatients, to improve the care of patients with both long term conditions and those with acute problems. Reference was also made to improving patients' experience of care.

In paediatrics, these visions are most easily realised within ambulatory paediatrics. In April 2014 a pioneering ambulatory care service was established at our hospital, with 'Healthcare at Home' (HAH). The first childrens' service of its kind, it is a consultant led, nurse delivered model of acute paediatric care. The nurses visit children up to four times a day, to administer medication, perform observations and provide clinical review. The observations and notes are recorded electronically. A daily consultant-led virtual ward round is conducted with the HAH nurses, facilitated by review of the patient's electronic health records. The initial goal was to enable early discharge from hospital, with future aspirations to facilitate admission avoidance with direct admission to HAH from the paediatric emergency department.

Methods Activity data for the first 9 months has been analysed and patient experience feedback has been evaluated.

Results 78 patients have been accepted onto the HAH service to date, giving 815 visits, and saving 389 bed days. 796/815 $(97.7 \%)$ were for administration of IV medication, with $48 \%$ for medications needing to be administered more than once a day. Referrals are increasing, with the majority from the general paediatrics service $(90.5 \%)$. Orthopaedics, gastroenterology and neurosurgery contribute the remainder. There have been 28 episodes of patients re-attending following transfer to $\mathrm{HAH} ; 22 / 28$ $(78.6 \%)$ have been due to problems with intravenous access, and $6 / 28$ (21.4\%) for clinical review. No patients have required readmission to the hospital. Patient feedback has been excellent with $100 \%$ of questionnaire responders saying they would recommend HAH to their friends and relatives.

Conclusion HAH is in its infancy but, thus far, has delivered exemplary clinical care. As stated in the NHS mandate, improving the experience of patients' and their families is essential The development of a flexible and robust community nursing service, which provides excellent acute clinical care is a proven means of facilitating this.

\section{G291 THE FRIENDS AND FAMILY TEST FOR CHILDREN AND YOUNG PEOPLE: KEY RECOMMENDATIONS}

${ }^{1} \mathrm{~A}$ Tallett, ${ }^{1} \mathrm{~A}$ Chisholm, ${ }^{1} \mathrm{~B}$ Hopwood, ${ }^{2} \mathrm{~S}$ Summersgill, ${ }^{2} \mathrm{~S}$ Manning. ${ }^{1}$ Health Experiences Team, Picker Institute Europe, Oxford, UK; ${ }^{2}$ Royal London Hospital, Barts Health NHS Trust, London, UK

\subsection{6/archdischild-2015-308599.268}

Aims The Friends and Family Test (FFT) is currently used nationally in adult acute care settings, and from April 2015 it will be compulsory for all children and young people (or their parents) to be offered the question. Although the guidance suggests wording for younger patients, there is no evidence that it has been rigorously tested with them. Our research therefore explored children's views of the FFT question including wording, response options and overall design, to propose the most suitable version for children and young people.

Methods A focus group with young patients explored views on patient feedback and different versions of the FFT. This informed the development of several child-friendly versions of the question, differing in wording and style. Eight interviews were conducted with patients at a large acute hospital trust, and their preferred wording and presentation options were incorporated into a final survey form. This was cognitively tested with twelve young patients ranging from four to fifteen years old, to ensure that questions were appropriately worded and interpreted as intended.

Results The adult question phrasing was unpopular: the word "recommend" and the response options "likely" and "unlikely" were problematic and not always understood. The phrase "if they needed similar care or treatment to me" was essential for understanding of the question. A five-scale response scale was recommended over a three-scale option as it ensured that a range of responses were used. Children preferred to feedback using an "agree/disagree" scale, and favouredimages of a monkey character over cartoons and easy-read style photos.

Conclusion This research highlights the importance of involving children and young people in the design and wording of questions to ensure that they are appealing and appropriate to them. The question form is currently being piloted with two large acute NHS Trusts using a hand-out methodology with an online option. We encourage healthcare organisations to adopt our format when implementing the FFT for their paediatric patients, since it has been thoroughly tested to ensure that it is suitable for younger populations.

\section{G292 ARE WE DOING IT RIGHT? COUNSELLING FOR RADIOLOGICAL IMAGING IN NON-ACCIDENTAL INJURY}

${ }^{1} E$ Payne, ${ }^{2} E$ Morris, ' J Natarajan. ${ }^{1}$ Paediatrics, Cwm Taf University Health Board, Llantrisant, UK; ${ }^{2}$ Radiology, CWm Taf University Health Board, Llantrisant, UK

\subsection{6/archdischild-2015-308599.269}

Aims Children undergoing radiological investigations for child protection are exposed to radiation. Good communication is vital if the child is to be properly and safely investigated. A requirement of obtaining informed consent for these investigations is to understand and be able to explain the risk / benefit of the procedures. We aim to identify how this is carried out nationwide and discuss attitudes and practice around full informed consent in child protection imaging.

Methods We carried out an online survey of all paediatric doctors in the UK. The survey was advertised in the RCPCH Wales e-bulletin and the RCPCH Health Policy bulletin.

Results From Wales and England, 61 paediatric doctors completed the survey. Of which, $44 \%$ of responders were consultants. Consent is taken for radiological imaging by $67 \%$. Verbal consent only is taken by $58 \%$. Only $36 \%$ provide information on the radiation exposure caused by the radiological imaging. Despite this, $80 \%$ of responders thought it was important to explain the radiation risk of doing such investigations.

Conclusion Although paediatricians consider it important to explain the risk/benefit of radiological procedures for child protection imaging, less than half undertake full written informed consent with fewer consenting for specific radiation exposure risks. Is this reluctance to take full informed consent due to a 